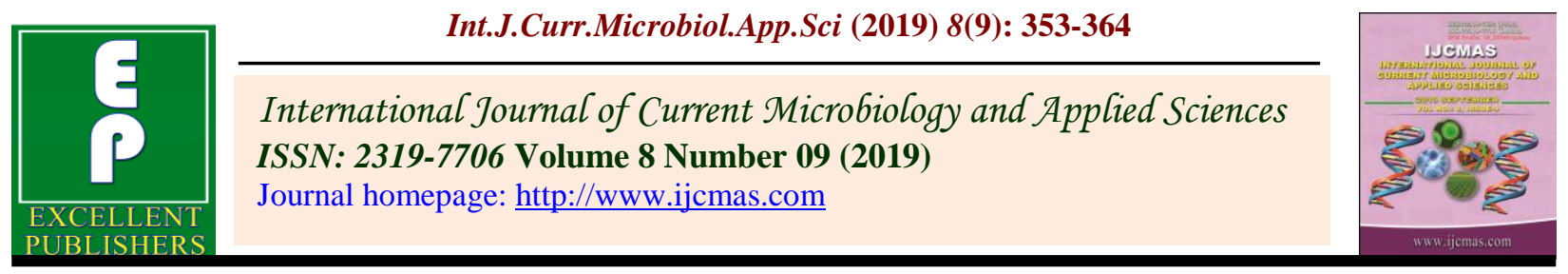

Original Research Article

https://doi.org/10.20546/ijcmas.2019.809.042

\title{
Studies on Combining Ability for Yield and Its Component Traits in Rabi Sorghum [Sorghum bicolor (L.) Moench]
}

\author{
N. Sandeep, B.D. Biradar*, Mruthunjaya C. Wali and R.A. Balikai \\ Department of Genetics and Plant Breeding \\ University of Agricultural Sciences, Dharwad - 580 005, Karnataka, India \\ *Corresponding author
}

\section{A B S T R A C T}

\begin{abstract}
Keywords
line $\times$ tester, GCA,

SCA, Combining

ability, Rabi

sorghum

Article Info

Accepted:

04 August 2019

Available Online:

10 September 2019

The study was carried out to assess the general combining ability of the parents and specific combining ability of the hybrids, using line $\mathrm{x}$ tester mating design. Fifty hybrids (derived from mating two testers with twenty-five lines in $\mathrm{L} x \mathrm{~T}$ design) along with their parents and checks (M35-1, BJV 44 and PKV Kranti) were evaluated during the rabi 2018-19. The ratio of $\sigma^{2} \mathrm{GCA} / \sigma^{2}$ SCA was less than unity for all the characters except for panicle indicating preponderance of non-additive gene action. Among female parents $M$ 31-2A was the best combiner for grain yield per plant, number of seeds per plant and 100 seed weight and 104A for panicle length. Among male parents, IS 995, IS 19450, IS 23590 and IS 23891 were found to be the best general combiner for grain yield per plant and number of seeds per panicle. The cross combinations viz., M 31-2A × IS 11619 and M 31$2 \mathrm{~A} \times$ IS 32439 exhibited highest and positive $s c a$ effects for grain yield per plant.
\end{abstract}

\section{Introduction}

Sorghum [Sorghum bicolor (L.) Moench] is one of the important cereal crop cultivated globally for food, fodder, feed and fuel. It ranks fifth after wheat, rice, maize and barley in area and production. It is the second cheapest source of energy and micronutrient after pearl millet. It is mainly grown in semiarid tropics of Asia, Africa, America and Australia, In Africa and Asia sorghum grain is mainly used as food, while in the United States and Australia it is used to feed cattle (Reddy et al., 2013). Globally, sorghum is grown in an area of 42.50 million hectares to produce 59.91 million tonnes, with the productivity of around 1.60 tonnes per hectare.

Sorghum has wide range of adaptability to various agroecological situations of the region. Combining ability studies provide useful information regarding the selection of suitable parents for effective hybridization programme (Sprague and Tatum, 1942). It also indicates the nature and magnitude of various types of gene action involved in the expression of quantitative characters. Such information is of potential use in formulating and executing an efficient breeding programme for achieving maximum genetic gain with minimum 
resources and time. Hence, this study was conducted with the aim to estimate the combining ability of the selected lines and testers in sorghum.

\section{Materials and Methods}

The male sterile lines viz., 104A and M 31-2A representing milo and maldandi sources of male sterility, respectively were used as testers. Twenty-five diverse germplasm accessions were selected from minicore collection and were crossed to each of these two male sterile lines in line $\mathrm{x}$ tester fashion (Kempthorne, 1957) to obtain 50 hybrids. These 50 hybrids along with their parents and three checks viz., M-35-1, BJV 44 and PKV Kranti were grown in randomized block design during rabi 2018-19 at Dharwad. The hybrids were grown each in a single row of four meters length in three replications with spacing of $45 \mathrm{~cm} \times 15 \mathrm{~cm}$ and all the recommended agronomic practises are followed to raise the good crop.

Biometrical observations was recorded on five randomly selected competitive plants in each replication for the traits viz., days to 50 per cent flowering, plant height $(\mathrm{cm})$, number of leaves per plant, panicle length $(\mathrm{cm})$, panicle width $(\mathrm{cm})$, number of primaries per panicle, number of seeds per panicle, panicle weight (g), 100 seed weight (g) and grain yield per plant $(\mathrm{g})$. The mean values of observations were subjected to statistical analysis to estimate general combining ability ( $g c a$ ) and specific combining ability ( $s c a$ ) effects of the parents and crosses (Panse and Sukhatme, 1967).

\section{Results and Discussion}

The analysis of combining ability variance for yield and yield related traits is presented in Table 1. The mean sum of squares due to crosses was found to be significant for all the traits. The mean sum of squares due to lines was significant for all the traits except for number of primaries per panicle. The traits like plant height, number of leaves, panicle length, panicle weight, grain yield per plant and 100 seed weight have shown peak significant mean sum of squares among the testers. The line $\times$ tester effects also shown significant mean sum of squares for all the traits.

The per cent contribution of lines, testers and line $\times$ tester along with estimates of GCA and SCA variance are presented in Table 1 . The magnitude of SCA variance was higher than GCA variance for all the nine traits studied except for panicle length (Jadhav and Deshmukh 2017) indicating the presence of non-additive variance and non-additive gene action indicates preponderance of non-additive gene action. Prevalence of non-additive gene action in sorghum was reported by Kumar and Chand (2015) and Dehiwal et al., (2017).

It is evident from the table that lines revealed preponderance influencer in the expression all traits studied viz., days to 50 per cent flowering ( $77.50 \%)$, plant height ( $57.87 \%)$, number of leaves per plant $(74.12 \%)$, number of primaries per panicle $(53.52 \%)$, panicle length $(52.32 \%)$, panicle width (53.79\%), panicle weight $(58.93 \%)$, grain yield per plant $(51.69 \%)$, number of seeds per panicle (71.54 $\%)$ and 100 seed weight $(71.52 \%)$.

\section{General combining ability (gca) effects}

Significant and negative gca effects are desirable for days to 50 per cent flowering. Out of twenty-five lines, twenty-three lines showed significant $\mathrm{gca}$ effect for days to 50 per cent flowering. However, eleven were in negative and twelve were in positive direction. IS 19975 (14.03) and IS 26025 (6.83) accounted for high $\mathrm{gca}$ in negative direction which is desirable. None of the testers was found to be significant in either positive or negative direction for gca effect (Table 2). 
As regards to plant height the magnitude of variation for $\mathrm{gca}$ effects for lines ranged from 17.50 (IS 30451) to -29.32 (IS 26617). The lines viz., IS 30451 (17.50) and IS 4581 (17.30) were found to have positive significance. Extreme negative significant gca effect was indexed by the line IS 26617. In case of testers, M 31-2A (5.24) had positive significance. With respect to number of leaves per plant estimates of $g c a$ were significant for 15 lines and out of which seven were found to be in positive direction. The lines viz., IS 31651 (2.92), IS 30451 (2.08) and IS 11619 (1.92) and tester M 31-2A (0.54) were found having significant gca positive effect.

The estimates of gca effects for panicle width varied from -0.93 (IS 19450) to 0.90 (IS 26025). Out of 25 lines, 16 lines indexed for significant $g \mathrm{ca}$ effects. Of all these, the lines viz., IS 26025 (0.90), IS 30451 (0.78), IS 25989 (0.68) and IS 24462 (0.65) have accounted for highest $\mathrm{gca}$ effect for panicle width. The magnitude of variation of $\mathrm{gca}$ effects for panicle length among lines varied from 9.41 (IS 602) to -5.41 (IS 11619). Out of 25 lines, 14 exhibited significant gca effects, seven lines exhibited significant positive $\mathrm{gca}$ effect in positive direction. The line viz., IS 602 (9.41) accounted for highest significant gca effect in positive direction. Among testers 104A (2.26) had positive significance.

Among 25 lines, 20 lines accounted for significant gca effect for number of primaries per panicle. Out of 9 lines which were showing positive gca effects the lines viz., IS 19450 (13.73) and IS 29269 (9.23) accounted for highest significant $\mathrm{gca}$ effect in positive direction. Among testers, M 31-2A (1.78) was found positively significant.

The range of $g c a$ effects for panicle weight for lines and testers varied from -21.88 (IS 20679) to 26.83 (IS 32439) and -4.62 (104A) to 4.62 (M 31-2A), respectively. Among lines, IS
32439 (26.83) and IS 19450 (25.36) showed peak positive significant gca effect. In contrast, the lines IS 20679 (21.85) and IS 602 (18.50) showed peak negative significant gca effect. Among testers, M 31-2A exhibited positive significant $\mathrm{gca}$ effect.

Grain yield per plant is very important yield contributing trait Among lines, the magnitude of variation of gca effects for grain yield per plant varied from -13.13 (IS 20679) to 13.58 (IS 19450). The lines viz., IS 19450 (13.58), IS 23590 (11.94) and IS 23891(11.39) accounted for highest significant positive $\mathrm{gca}$ effects. Among testers, M 31-2A (3.79) exhibited significant positive gca effect. For number of seeds per panicle the $g c a$ effects among lines varied between -561.88 (IS 4581) to 819.94 (IS 23891).

Nine lines recorded significant $\mathrm{gca}$ effects in positive direction. The line IS 23891 recorded highest significant positive gca effects followed by IS 995 (722.11). On the other hand the lines IS 4581 (561.88) and IS 29654 (538.72) recorded highest significant negative gca effects. Among testers, M 31-2A (76.56) exhibited significant positive $g c a$ effects.

Among lines, the estimates of gca effect for hundred seed weight varied from -0.63 (IS 23891 ) to 1.23 (IS 4581). Out of 25 lines, eight were found to have significant effect for gca. The line IS 4581 (1.29) topped the list showing positive gca effect, whereas the line IS 23891 (0.63) exhibited highest significant gca in negative direction. The tester M 31-2A (0.10) recorded significant positive gca effect.

\section{Specific combining ability (sca) effects}

Among 50 crosses, 15 crosses exhibited significant sca effects in negative desirable direction for days to 50 per cent flowering. 
Table.1 ANOVA for combining ability for yield and yield components in rabi sorghum

\begin{tabular}{|c|c|c|c|c|c|c|}
\hline \multirow[t]{2}{*}{ Source of variation } & & 1 & 2 & 3 & 4 & 5 \\
\hline & df & $\begin{array}{l}\text { Days to } 50 \% \\
\text { flowering }\end{array}$ & Plant height(cm) & $\begin{array}{c}\text { Number of leaves } \\
\text { per plant }\end{array}$ & $\begin{array}{c}\text { Number of primaries } \\
\text { panicle }^{-4}\end{array}$ & Panicle length $(\mathrm{cm})$ \\
\hline Replication & 2 & 0.88 & 1.047 & 0.060 & 0.34 & 5.87 \\
\hline Crosses & 49 & $103.52 * *$ & $590.41 * *$ & $8.44 * *$ & $163.44 * *$ & $63.91 * *$ \\
\hline Lines effect & 24 & $163.81 * *$ & $676.60 *$ & $12.77 * *$ & 178.62 & $68.21 *$ \\
\hline Testers effect & 1 & 2.16 & $4129.12 * *$ & $44.82 * *$ & 478.82 & $770.16 * *$ \\
\hline Lines $\times$ Testers effect & 24 & $47.45 * *$ & $335.77 * *$ & $2.59 * *$ & $135.11 * *$ & $30.11 * *$ \\
\hline Error & 98 & 2.41 & 19.94 & 0.64 & 3.40 & 6.26 \\
\hline Total & 149 & 35.64 & 207.29 & 3.20 & 55.99 & 25.14 \\
\hline$\sigma^{2}$ GCA & & $2.00 * *$ & $59.21 * *$ & $0.700 * *$ & $8.03 *$ & $10.24 * *$ \\
\hline$\sigma^{2} \mathrm{SCA}$ & & $15.17 * *$ & $106.92 * *$ & $0.717 * *$ & $43.89 * *$ & $8.64 * *$ \\
\hline$\sigma^{2} \mathrm{GCA} / \sigma^{2} \mathrm{SCA}$ & & 0.13 & 0.55 & 0.98 & 0.18 & 1.18 \\
\hline Contribution (\%) of Lines & & 77.50 & 57.87 & 74.13 & 53.52 & 52.32 \\
\hline Contribution (\%) of Testers & & 0.04 & 14.27 & 10.83 & 5.97 & 24.59 \\
\hline $\begin{array}{l}\text { Contribution }(\%) \text { of Line } \times \\
\text { Tester }\end{array}$ & & 22.45 & 27.85 & 15.02 & 40.49 & 23.07 \\
\hline \multirow[t]{2}{*}{ Source of variation } & & 6 & 7 & 8 & 9 & 10 \\
\hline & df & $\begin{array}{l}\text { Panicle width } \\
(\mathrm{cm})\end{array}$ & $\begin{array}{c}\text { Panicle Weight } \\
\text { (g) }\end{array}$ & $\begin{array}{l}\text { Grain yield per } \\
\text { plant (g) }\end{array}$ & $\begin{array}{c}\text { Number of seeds per } \\
\text { panicle }\end{array}$ & $\begin{array}{l}100 \text { Seed weight } \\
\text { (g) }\end{array}$ \\
\hline Replication & 2 & 0.11 & 1.44 & 14.59 & 10874.33 & 0.08 \\
\hline Crosses & 49 & $1.35^{* *}$ & $789.60 * *$ & $336.02 * *$ & $570629.30 * *$ & $0.80 * *$ \\
\hline Lines effect & 24 & 1.49 & 950.08 & 354.62 & $833580.90 * *$ & $1.17 * *$ \\
\hline Testers effect & 1 & 0.12 & $3213.68 * *$ & $2158.76^{* *}$ & 879215.10 & $1.77 *$ \\
\hline Lines $\times$ Testers effect & 24 & $1.27 * *$ & $528.12 * *$ & $241.48 * *$ & $294819.90 * *$ & $0.39 * *$ \\
\hline Error & 98 & 0.06 & 0.52 & 5.10 & 8155.11 & 0.07 \\
\hline Total & 149 & 0.49 & 260.03 & 114.06 & 193166.30 & 0.31 \\
\hline$\sigma^{2} \mathrm{GCA}$ & & 0.01 & $51.37 * *$ & $30.89 * *$ & $20859.91 * *$ & $0.03 * *$ \\
\hline$\sigma^{2} \mathrm{SCA}$ & & $0.40 * *$ & $175.70 * *$ & $78.68 * *$ & $94416.16 * *$ & $0.11 * *$ \\
\hline$\sigma^{2} \mathrm{GCA} / \sigma^{2} \mathrm{SCA}$ & & 0.025 & 0.29 & 0.39 & 0.21 & 0.27 \\
\hline Contribution (\%) of Lines & & 53.79 & 58.93 & 51.69 & 71.54 & 71.52 \\
\hline Contribution (\%) of Testers & & 0.19 & 8.03 & 13.11 & 3.14 & 4.50 \\
\hline $\begin{array}{l}\text { Contribution }(\%) \text { of Line } \times \\
\text { Tester }\end{array}$ & & 46.07 & 32.75 & 35.19 & 25.30 & 23.97 \\
\hline
\end{tabular}

*** significant at 5 and 1 per cent respectively 
Table.2 Estimates of general combining ability effects of parents for yield and its attributing traits in rabi sorghum

\begin{tabular}{|c|c|c|c|c|c|c|c|c|c|c|c|}
\hline $\begin{array}{l}\text { Sl. } \\
\text { No. }\end{array}$ & Parents & $\begin{array}{c}\text { Days to } 50 \\
\text { per cent } \\
\text { flowering }\end{array}$ & $\begin{array}{l}\text { Plant } \\
\text { height }\end{array}$ & $\begin{array}{c}\text { Number } \\
\text { of } \\
\text { leaves }\end{array}$ & $\begin{array}{l}\text { Panicle } \\
\text { length }\end{array}$ & $\begin{array}{l}\text { Panicle } \\
\text { width }\end{array}$ & $\begin{array}{l}\text { Primaries } \\
\text { panicle }^{-1}\end{array}$ & $\begin{array}{c}\text { Panicle } \\
\text { weight }\end{array}$ & $\begin{array}{c}\text { Number of } \\
\text { seeds } \\
\text { panicle }^{-1}\end{array}$ & $\begin{array}{l}\text { Grain } \\
\text { yield } \\
\text { plant }^{-1}\end{array}$ & $\begin{array}{c}100 \text { seed } \\
\text { weight }\end{array}$ \\
\hline & Testers & & & & & & & & & & \\
\hline 1 & 104B & -0.12 & $-5.24 * *$ & $-0.54 * *$ & $2.26 * *$ & 0.029 & $-1.78 * *$ & $-4.62 * *$ & $-76.56 * *$ & $-3.79 * *$ & $-0.109 * *$ \\
\hline \multirow[t]{3}{*}{2} & M 31-2B & 0.12 & $5.24 * *$ & $0.54 * *$ & $-2.26 * *$ & -0.029 & $1.78^{* *}$ & $4.62 * *$ & $76.56 * *$ & $3.79 * *$ & $0.109 * *$ \\
\hline & $\mathrm{CD}$ at $5 \%$ & 0.31 & 0.88 & 0.15 & & 0.05 & & & & 0.53 & 0.05 \\
\hline & Lines & & & & & & & & & & \\
\hline 3 & IS 602 & $-3.03 * *$ & $-7.87 * *$ & $-1.58 * *$ & $9.41 * *$ & -0.14 & $-10.43 * *$ & $-18.50 * *$ & 63.13 & $-6.08 * *$ & $-0.59 * *$ \\
\hline 4 & IS 995 & $-4.70 * *$ & $13.17 * *$ & $-3.08 * *$ & $5.36 * *$ & $0.36 * *$ & $3.90 * *$ & $10.60 * *$ & $722.11 * *$ & $10.26 * *$ & $-0.50 * *$ \\
\hline 5 & IS 4515 & $5.46 * *$ & 1.67 & $-1.58 * *$ & $-3.04 * *$ & -0.17 & -0.10 & $1.06^{*}$ & $-497.55 * *$ & $-11.43 * *$ & 0.16 \\
\hline 6 & IS 4581 & $7.36 * *$ & $17.30 * *$ & 0.25 & & & $6.56 * *$ & $-10.50 * *$ & $-561.88 * *$ & $-6.21 * *$ & $1.29 * *$ \\
\hline 7 & IS 4698 & -1.03 & -1.66 & $-1.58 * *$ & $-4.41 * *$ & $-0.29 * *$ & $2.06 * *$ & $2.53 * *$ & $108.44^{*}$ & 1.44 & $-0.22 *$ \\
\hline 8 & IS 11619 & $7.636 * *$ & $15.00 * *$ & $1.92 * *$ & $-5.41 * *$ & -0.03 & $-3.26 * *$ & $7.54 * *$ & $-163.88 * *$ & 0.81 & $0.19 *$ \\
\hline 9 & IS 15945 & $6.96 * * *$ & $7.50 * *$ & $1.42 * *$ & $3.73 * *$ & 0.11 & $-3.60 * *$ & $7.54 * *$ & $258.44 * *$ & -0.28 & $-0.54 * *$ \\
\hline 10 & IS 19450 & $3.13 * *$ & $-4.32 * *$ & $-2.58 * *$ & -1.61 & $0.90 * *$ & $13.73 * *$ & $25.36 * *$ & $436.94 * *$ & $13.58 * *$ & -0.02 \\
\hline 11 & IS 19975 & $-14.03 * *$ & $-4.82 * *$ & $0.92 * *$ & $2.55 * *$ & -0.13 & $-7.43 * *$ & $-6.67 * *$ & $-164.72 * *$ & $-4.50 * *$ & -0.07 \\
\hline 12 & IS 20743 & $-1.20 *$ & -0.99 & 0.25 & $-2.64 * *$ & $-0.66 * *$ & $-1.93 *$ & $-2.20 * *$ & $262.94 * *$ & 1.46 & $-0.42 * *$ \\
\hline 13 & IS 20679 & $-1.36^{*}$ & $-7.99 * *$ & 0.42 & -0.64 & $-0.74 * *$ & $-2.43 * *$ & $-21.85^{* *}$ & $-521.88 * *$ & $-13.13 * *$ & 0.09 \\
\hline 14 & IS 22720 & $-1.70 * *$ & $-4.49 * *$ & $-1.58 * *$ & $-1.89 * *$ & -0.08 & 0.40 & $-12.67 * *$ & $-377.88 * *$ & $-8.76^{* *}$ & 0.09 \\
\hline 15 & IS 23590 & $3.96 * *$ & $12.84 * *$ & $0.75 * *$ & -1.61 & $0.51 * *$ & 1.23 & $11.59 * *$ & $503.94 * *$ & $11.94 * *$ & $-0.20^{*}$ \\
\hline 16 & IS 23891 & -0.96 & $-6.82 * *$ & 0.25 & 0.23 & $031 * *$ & 1.40 & $16.44 * *$ & $819.94 * *$ & $11.39 * *$ & $-0.63 * *$ \\
\hline 17 & IS 24462 & $1.53 * *$ & 2.00 & -0.24 & $2.35 *$ & $0.65 * *$ & 1.40 & -0.05 & 82.11 & 1.11 & -0.05 \\
\hline
\end{tabular}




\begin{tabular}{|c|c|c|c|c|c|c|c|c|c|c|c|c|}
\hline $\begin{array}{l}\text { Sl. } \\
\text { No. }\end{array}$ & Parents & $\begin{array}{c}\text { Days to } 50 \\
\text { per cent } \\
\text { flowering }\end{array}$ & $\begin{array}{l}\text { Days to } \\
\text { maturity }\end{array}$ & $\begin{array}{l}\text { Plant } \\
\text { height }\end{array}$ & $\begin{array}{c}\text { Number } \\
\text { of } \\
\text { leaves }\end{array}$ & $\begin{array}{l}\text { Panicle } \\
\text { length }\end{array}$ & $\begin{array}{c}\text { Panicle } \\
\text { width }\end{array}$ & $\begin{array}{l}\text { Primaries } \\
\text { panicle }^{-1}\end{array}$ & $\begin{array}{c}\text { Panicle } \\
\text { weight }\end{array}$ & $\begin{array}{l}\text { Number } \\
\text { of seeds } \\
\text { panicle }^{-1}\end{array}$ & $\begin{array}{l}\text { Grain } \\
\text { yield } \\
\text { plant }^{-1}\end{array}$ & $\begin{array}{c}100 \\
\text { seed } \\
\text { weight }\end{array}$ \\
\hline 18 & IS 25989 & $-1.94 * *$ & $-2.32 * *$ & 2.34 & $1.58 * *$ & 1.03 & $0.68 * *$ & $6.56 * *$ & $4.51 * *$ & $152.44 * *$ & 1.61 & $-0.22 *$ \\
\hline 19 & IS 26025 & $-6.83 * *$ & $-6.98 * *$ & $-12.87 * *$ & -0.08 & $2.75 * *$ & $-0.93 * *$ & $-4.43 * *$ & $-6.32 * *$ & 6.61 & $-3.06 * *$ & $-0.27 * *$ \\
\hline 20 & IS 26617 & $1.13^{*}$ & $3.18 * *$ & $-29.32 * *$ & $-0.58 *$ & -1.09 & $-0.58 * *$ & $-4.43 * *$ & $-9.27 * *$ & $-132.05 * *$ & $-2.25^{*}$ & 0.02 \\
\hline 21 & IS 27887 & $-5.53 * *$ & $-5.48 * *$ & -0.99 & -0.08 & $-2.06^{*}$ & $0.40 * *$ & $1.56^{*}$ & $-14.68 * *$ & $-277.05 * *$ & $-6.30 * *$ & 0.04 \\
\hline 22 & IS 28313 & $3.96 * *$ & $2.51 * *$ & 2.00 & 0.42 & -1.18 & $-0.59 * *$ & $-6.26 * *$ & $-2.42 * *$ & $-154.22 * *$ & -0.43 & $0.27 * *$ \\
\hline 23 & IS 29269 & $-3.86^{* *}$ & $-4.98 * *$ & $4.67 * *$ & 0.42 & -1.38 & 0.05 & $9.23 * *$ & $5.01 * *$ & $-233.38 * *$ & 1.04 & $0.57 * *$ \\
\hline 24 & IS 29654 & $8.63 * *$ & $6.84 * *$ & $-4.49 * *$ & $-1.08 * *$ & $-4.88 * *$ & $-0.31 * *$ & $-1.60 *$ & $-15.85^{* *}$ & $-538.72 * *$ & $-11.26 * *$ & $0.39 * *$ \\
\hline 25 & IS 30451 & $-1.20 *$ & $1.18^{*}$ & $17.50 * *$ & $2.08 * *$ & 0.16 & $0.78 * *$ & $1.56^{*}$ & $3.36 * *$ & 23.28 & $9.98 * *$ & $0.61 * *$ \\
\hline 26 & IS 31651 & $-3.70 * *$ & $-3.65 * *$ & $-14.66 * *$ & $2.92 * *$ & 1.25 & -0.16 & $-6.26 * *$ & $-1.42 * *$ & $119.78 * *$ & -0.36 & $-0.39 * *$ \\
\hline \multirow[t]{2}{*}{27} & IS 32439 & $1.13^{*}$ & 1.01 & $5.17 * *$ & 0.42 & $3.60 * *$ & -0.16 & $2.56^{* *}$ & $26.83 * *$ & 63.11 & $9.42 * *$ & $0.39 * *$ \\
\hline & C.D @ 5\% & 1.12 & 1.03 & 3.13 & 0.70 & 1.65 & 0.19 & 1.50 & 0.81 & 87.14 & 1.88 & 0.17 \\
\hline
\end{tabular}

$*$ and **indicates significant at 5 and 1 per cent, respectively. 
Table.3a Estimates of specific combining ability effects of crosses for yield and its attributing traits in rabi sorghum

\begin{tabular}{|c|c|c|c|c|c|c|}
\hline $\begin{array}{l}\text { Sl. } \\
\text { No. }\end{array}$ & Crosses & $\begin{array}{c}\text { Days to } 50 \text { per cent } \\
\text { flowering }\end{array}$ & Plant height & No of leaves & Panicle length & $\begin{array}{c}\text { Panicle } \\
\text { width }\end{array}$ \\
\hline 1 & $104 \mathrm{~A} \times \mathrm{IS} 602$ & $3.95 * *$ & $8.74 * *$ & 0.04 & -2.01 & -0.22 \\
\hline 2 & $104 \mathrm{~A} \times \mathrm{IS} 995$ & $2.62 * *$ & $-16.92 * *$ & 0.54 & $-4.83 * *$ & 0.12 \\
\hline 3 & $104 \mathrm{~A} \times \mathrm{IS} 4515$ & $-2.88 * *$ & 2.91 & 0.04 & 2.08 & 0.14 \\
\hline 4 & $104 \mathrm{~A} \times \mathrm{IS} 4581$ & -1.38 & 0.91 & -0.12 & 0.45 & $0.40^{* *}$ \\
\hline 5 & $104 \mathrm{~A} \times \mathrm{IS} 4698$ & 0.28 & $12.58 * *$ & 0.04 & -2.08 & $-0.27^{*}$ \\
\hline 6 & $104 \mathrm{~A} \times \mathrm{IS} 11619$ & 0.95 & -1.42 & 0.54 & -0.49 & 0.04 \\
\hline 7 & $104 \mathrm{~A} \times \mathrm{IS} 15945$ & $-1.71 *$ & $-4.58 *$ & 0.04 & -2.03 & $-0.82 * *$ \\
\hline 8 & $104 \mathrm{~A} \times \mathrm{IS} 19450$ & 0.78 & $8.91 * *$ & 0.04 & $5.03 * *$ & $0.35 * *$ \\
\hline 9 & $104 \mathrm{~A} \times \mathrm{IS} 19975$ & 0.28 & 1.74 & $1.54 * *$ & -0.81 & $0.45 * *$ \\
\hline 10 & $104 \mathrm{~A} \times \mathrm{IS} 20743$ & -1.54 & 0.24 & $-0.78 *$ & 0.48 & $0.38 * *$ \\
\hline 11 & $104 \mathrm{~A} \times \mathrm{IS} 20679$ & -0.04 & -4.42 & 0.04 & -0.31 & -0.09 \\
\hline 12 & $104 \mathrm{~A} \times \mathrm{IS} 22720$ & $2.28 * *$ & -2.25 & 0.04 & 1.60 & -0.02 \\
\hline 13 & $104 \mathrm{~A} \times \mathrm{IS} 23590$ & 0.28 & $6.41 * *$ & -0.28 & 1.01 & $0.63 * *$ \\
\hline 14 & $104 \mathrm{~A} \times \mathrm{IS} 23891$ & $-5.21 * *$ & -1.58 & $-0.78 *$ & -0.86 & 0.03 \\
\hline 15 & $104 \mathrm{~A} \times \mathrm{IS} 24462$ & $5.28 * *$ & $6.24 * *$ & -0.28 & -0.26 & $0.40 * *$ \\
\hline 16 & $104 \mathrm{~A} \times \mathrm{IS} 25989$ & -0.21 & -1.75 & 0.54 & -0.83 & -0.02 \\
\hline 17 & $104 \mathrm{~A} \times \mathrm{IS} 26025$ & -0.54 & $-8.25 * *$ & -0.45 & -1.83 & $0.48 * *$ \\
\hline 18 & $104 \mathrm{~A} \times \mathrm{IS} 26617$ & $2.78 * *$ & $-9.08 * *$ & 0.04 & $4.33 * *$ & $0.33 * *$ \\
\hline 19 & $104 \mathrm{~A} \times \mathrm{IS} 27887$ & $-2.21 * *$ & -0.42 & -0.45 & 1.30 & -0.01 \\
\hline 20 & $104 \mathrm{~A} \times \mathrm{IS} 28313$ & $-6.38 * *$ & -1.42 & 0.04 & -1.04 & 0.02 \\
\hline 21 & $104 \mathrm{~A} \times \mathrm{IS} 29269$ & 1.45 & 2.91 & 0.04 & 1.68 & $-0.56 * *$ \\
\hline 22 & $104 \mathrm{~A} \times \mathrm{IS} 29654$ & -0.71 & $7.41 * *$ & $-1.45^{* *}$ & 1.31 & 0.23 \\
\hline 23 & $104 \mathrm{~A} \times \mathrm{IS} 30451$ & $-3.21 * *$ & -0.58 & $1.38 * *$ & 0.03 & $-0.62 * *$ \\
\hline 24 & $104 \mathrm{~A} \times \mathrm{IS} 31651$ & 0.28 & $10.24 * *$ & 0.54 & 1.98 & -0.01 \\
\hline 25 & $104 \mathrm{~A} \times \mathrm{IS} 32439$ & $4.78 * *$ & $-16.58 * *$ & $-0.95 *$ & $-3.83 * *$ & $-1.37 * *$ \\
\hline 26 & M 31-2A × IS 602 & $-3.95 * *$ & $-8.74 * *$ & -0.04 & 2.01 & 0.22 \\
\hline
\end{tabular}




\begin{tabular}{|c|c|c|c|c|c|c|}
\hline $\begin{array}{l}\text { Sl. } \\
\text { No. }\end{array}$ & Parents & $\begin{array}{c}\text { Days to } 50 \text { per cent } \\
\text { flowering }\end{array}$ & Plant height & No of leaves & Panicle length & $\begin{array}{c}\text { Panicle } \\
\text { width }\end{array}$ \\
\hline 27 & M 31-2A × IS 995 & $-2.62 * *$ & $16.92 * *$ & -0.54 & $4.83 * *$ & -0.12 \\
\hline 28 & M $31-2 A \times$ IS 4515 & $2.88 * *$ & -2.91 & -0.04 & -2.08 & -0.15 \\
\hline 29 & M $31-2 \mathrm{~A} \times \mathrm{IS} 4581$ & 1.38 & -0.91 & 0.12 & -0.45 & $-0.40 * *$ \\
\hline 30 & M $31-2$ A $\times$ IS 4698 & -0.28 & $-12.58 * *$ & -0.04 & 2.08 & $0.279 *$ \\
\hline 31 & M 31-2A $\times$ IS 11619 & -0.95 & 1.42 & -0.54 & 0.49 & -0.05 \\
\hline 32 & M 31-2A $\times$ IS 15945 & $1.71 *$ & $4.58 *$ & -0.04 & 2.03 & $0.83 * *$ \\
\hline 33 & M 31-2A × IS 19450 & -0.78 & $-8.91 * *$ & -0.04 & $-5.03 * *$ & $-0.36 * *$ \\
\hline 34 & M 31-2A × IS 19975 & -0.28 & -1.74 & $-1.54 * *$ & 0.81 & $-0.45 * *$ \\
\hline 35 & M 31-2A × IS 20743 & 1.54 & -0.27 & $0.78^{*}$ & -0.48 & $-0.38 * *$ \\
\hline 36 & M 31-2A × IS 20679 & 0.04 & 4.42 & -0.04 & 0.31 & 0.09 \\
\hline 37 & M $31-2 \mathrm{~A} \times$ IS 22720 & $-2.28 * *$ & 2.25 & -0.04 & -1.60 & 0.03 \\
\hline 38 & M 31-2A × IS 23590 & -0.28 & $-6.41 * *$ & 0.28 & -1.01 & $\begin{array}{c}- \\
0.637 * *\end{array}$ \\
\hline 39 & M 31-2A × IS 23891 & $5.21 * *$ & 1.58 & $0.78 *$ & 0.86 & -0.03 \\
\hline 40 & M 31-2A × IS 24462 & $-5.28 * *$ & $-6.24 * *$ & 0.28 & 0.26 & $-0.40 * *$ \\
\hline 41 & M 31-2A × IS 25989 & 0.21 & 1.75 & -0.54 & 0.83 & 0.03 \\
\hline 42 & M 31-2A × IS 26025 & 0.54 & $8.25 * *$ & 0.45 & 1.88 & $-0.48 * *$ \\
\hline 43 & M 31-2A × IS 26617 & $-2.78 * *$ & $9.08 * *$ & -0.04 & $-4.33 * *$ & $-0.33 *$ \\
\hline 44 & M 31-2A × IS 27887 & $2.21 * *$ & 0.42 & 0.45 & -1.30 & 0.01 \\
\hline 45 & M 31-2A $\times$ IS 28313 & $6.38 * *$ & 1.42 & -0.04 & 1.04 & -0.02 \\
\hline 46 & M 31-2A × IS 29269 & -1.45 & -2.91 & -0.04 & -1.68 & $0.56 * *$ \\
\hline 47 & M 31-2A × IS 29654 & 0.71 & $-7.41 * *$ & $1.45 * *$ & -1.31 & -0.23 \\
\hline 48 & M 31-2A × IS 30451 & $3.21 * *$ & 0.58 & $-1.38 * *$ & -0.03 & $0.62 * *$ \\
\hline 49 & M 31-2A × IS 31651 & 0.28 & $-10.24 * *$ & -0.54 & -1.98 & 0.01 \\
\hline \multirow[t]{2}{*}{50} & M 31-2A × IS 32439 & $-4.78 * *$ & $16.58 * *$ & $0.95^{*}$ & $3.83 * *$ & $1.37 * *$ \\
\hline & CD@ $5 \%$ & 1.59 & 4.43 & 0.75 & 2.34 & 0.26 \\
\hline
\end{tabular}

$*$ and $* *$ indicates significant at 5 and 1 per cent, respectively. 
Table.3b Estimates of specific combining ability effects of crosses for yield and its attributing traits in rabi sorghum

\begin{tabular}{|c|c|c|c|c|c|c|}
\hline $\begin{array}{l}\text { Sl. } \\
\text { No. }\end{array}$ & Crosses & Primaries panicle $^{-1}$ & Panicle weight & Grain yield per plant & $\begin{array}{l}\text { Number of seeds per } \\
\text { panicle }\end{array}$ & $\begin{array}{l}100 \text { seed } \\
\text { weight }\end{array}$ \\
\hline 1 & $104 \mathrm{~A} \times \mathrm{IS} 602$ & $-5.71 * *$ & $6.76 * *$ & $3.29 *$ & $242.39 * *$ & -0.19 \\
\hline 3 & $104 \mathrm{~A} \times \mathrm{IS} 4515$ & $2.95 * *$ & $-13.17 * *$ & $-3.82 * *$ & -77.27 & -0.21 \\
\hline 4 & $104 \mathrm{~A} \times \mathrm{IS} 4581$ & $-4.04 * *$ & $9.03 * *$ & $6.16^{* *}$ & $198.73 * *$ & $-0.37 * *$ \\
\hline 5 & $104 \mathrm{~A} \times \mathrm{IS} 4698$ & $5.79 * *$ & $-4.17 * *$ & $-5.34 * *$ & -111.61 & -0.16 \\
\hline 7 & $104 \mathrm{~A} \times \mathrm{IS} 15945$ & 2.12 & $-3.35 * *$ & 1.99 & $128.73^{*}$ & -0.07 \\
\hline 8 & $104 \mathrm{~A} \times \mathrm{IS} 19450$ & -1.55 & $-3.89 * *$ & -0.60 & 9.23 & -0.03 \\
\hline 9 & $104 \mathrm{~A} \times \mathrm{IS} 19975$ & $6.62 * *$ & $-16.64 * *$ & $-6.75 * *$ & $-287.11 * *$ & 0.09 \\
\hline 10 & $104 \mathrm{~A} \times \mathrm{IS} 20743$ & $5.12 * *$ & -0.32 & 0.17 & $176.56^{* *}$ & $-0.26^{*}$ \\
\hline 11 & $104 \mathrm{~A} \times \mathrm{IS} 20679$ & 0.28 & $10.18 * *$ & $7.24 * *$ & $137.39 *$ & $0.29 *$ \\
\hline 12 & $104 \mathrm{~A} \times \mathrm{IS} 22720$ & $-2.88 * *$ & $17.76 * *$ & $7.98 * *$ & $163.73 * *$ & $0.26^{*}$ \\
\hline 13 & $104 \mathrm{~A} \times \mathrm{IS} 23590$ & -0.71 & $1.96 * *$ & 2.43 & -7.77 & 0.16 \\
\hline 16 & $104 \mathrm{~A} \times \mathrm{IS} 25989$ & -1.38 & -0.65 & 0.96 & $-162.27 *$ & $0.34 * *$ \\
\hline 17 & $104 \mathrm{~A} \times \mathrm{IS} 26025$ & -0.71 & -0.02 & -0.89 & $-214.77 * *$ & $0.32 *$ \\
\hline 18 & $104 \mathrm{~A} \times \mathrm{IS} 26617$ & $6.29 * *$ & $1.29 *$ & 0.46 & 14.23 & -0.008 \\
\hline 19 & $104 \mathrm{~A} \times$ IS 27887 & $-4.71 * *$ & $11.48 * *$ & $7.84 * *$ & $136.89^{*}$ & $0.31 *$ \\
\hline 20 & $104 \mathrm{~A} \times \mathrm{IS} 28313$ & $2.45^{*}$ & $2.24 * *$ & $3.24 *$ & -71.61 & $0.44 * *$ \\
\hline 21 & $104 \mathrm{~A} \times \mathrm{IS} 29269$ & $5.28 * *$ & $-3.15 * *$ & -0.07 & 88.89 & $-0.26^{*}$ \\
\hline 22 & $104 \mathrm{~A} \times \mathrm{IS} 29654$ & 2.12 & $10.24 * *$ & $4.24 * *$ & $129.56^{*}$ & -0.04 \\
\hline 23 & $104 \mathrm{~A} \times \mathrm{IS} 30451$ & $-4.38 * *$ & $1.69 * *$ & 2.29 & -89.11 & $0.41 * *$ \\
\hline 24 & $104 \mathrm{~A} \times \mathrm{IS} 31651$ & $-10.55^{* *}$ & $-15.55 * *$ & $-9.05 * *$ & $-289.94 * *$ & -0.12 \\
\hline 25 & $104 \mathrm{~A} \times \mathrm{IS} 32439$ & $-8.71 * *$ & $-13.77 * *$ & $-12.31 * *$ & $-342.60 * *$ & -0.04 \\
\hline 26 & M 31-2A × IS 602 & $5.71 * *$ & $-6.76 * *$ & $-3.29 *$ & $-242.39 * *$ & 0.19 \\
\hline
\end{tabular}




\begin{tabular}{|c|c|c|c|c|c|c|}
\hline $\begin{array}{l}\text { Sl. } \\
\text { No. }\end{array}$ & Parents & $\begin{array}{c}\text { Primaries } \\
\text { panicle }^{-1}\end{array}$ & Panicle weight & Grain yield per plant & $\begin{array}{c}\text { Number of seeds per } \\
\text { panicle }\end{array}$ & $\begin{array}{c}100 \text { seed } \\
\text { weight }\end{array}$ \\
\hline 27 & M 31-2A × IS 995 & 0.38 & $5.13 * *$ & -0.68 & $-346.73 * *$ & $0.34 * *$ \\
\hline 28 & M $31-2 A \times I S 4515$ & $-2.95 * *$ & $13.17 * *$ & $3.82 * *$ & 77.27 & 0.21 \\
\hline 29 & M $31-2 \mathrm{~A} \times \mathrm{IS} 4581$ & $4.05 * *$ & $-9.02 * *$ & $-6.16^{* *}$ & $-198.73 * *$ & $0.37 * *$ \\
\hline 30 & M $31-2 A \times$ IS 4698 & $-5.79 * *$ & $4.17 * *$ & $5.34 * *$ & 111.61 & 0.16 \\
\hline 31 & M 31-2A $\times$ IS 11619 & 1.55 & $6.45 * *$ & $16.47 * *$ & $408.27 * *$ & $0.31 *$ \\
\hline 32 & M 31-2A × IS 15945 & -2.12 & $3.35^{* *}$ & -1.99 & $-128.73 *$ & 0.07 \\
\hline 33 & M 31-2A $\times$ IS 19450 & 1.55 & $3.83 * *$ & 0.60 & -9.23 & 0.02 \\
\hline 34 & M 31-2A × IS 19975 & $-6.62 * *$ & $16.64 * *$ & $6.76 * *$ & $287.11 * *$ & -0.09 \\
\hline 35 & M 31-2A × IS 20743 & $-5.12 * *$ & 0.32 & -0.19 & $-176.56^{* *}$ & $0.26^{*}$ \\
\hline 36 & M 31-2A × IS 20679 & -0.29 & $-10.18 * *$ & $-7.24 * *$ & $-137.39 * *$ & $-0.29 *$ \\
\hline 37 & M $31-2 A \times I S 22720$ & $2.88 * *$ & $-17.76^{* *}$ & $-7.98 * *$ & $-163.73 * *$ & $-0.26^{*}$ \\
\hline 38 & M 31-2A × IS 23590 & 0.71 & $-1.96 * *$ & -2.43 & 7.77 & -0.16 \\
\hline 39 & M 31-2A × IS 23891 & -1.12 & $3.69 * *$ & $3.12 *$ & $192.44 * *$ & -0.07 \\
\hline 40 & M $31-2 A \times$ IS 24462 & $-7.12 * *$ & $-17.28 * *$ & $-9.46 * *$ & $-481.73 * *$ & $0.29 *$ \\
\hline 41 & M 31-2A × IS 25989 & 1.38 & 0.65 & -0.96 & $162.27 *$ & $-0.34 * *$ \\
\hline 42 & M 31-2A × IS 26025 & 0.71 & 0.02 & 0.89 & $214.77 * *$ & $-0.32 *$ \\
\hline 43 & M 31-2A × IS 26617 & $-6.29 * *$ & $-1.29 *$ & -0.46 & -14.23 & 0.008 \\
\hline 44 & M 31-2A × IS 27887 & $4.71 * *$ & $-11.48 * *$ & $-7.84 * *$ & $-136.89 *$ & $-0.31 *$ \\
\hline 45 & M 31-2A $\times$ IS 28313 & $-2.45^{*}$ & $-2.24 * *$ & $-3.24 *$ & 71.61 & $-0.44 * *$ \\
\hline 46 & M 31-2A × IS 29269 & $-5.29 * *$ & $3.15^{* *}$ & 0.07 & -88.89 & $0.26^{*}$ \\
\hline 47 & M 31-2A × IS 29654 & -2.12 & $-10.2 * *$ & $-4.24 * *$ & $-129.56^{*}$ & 0.041 \\
\hline 48 & M 31-2A × IS 30451 & $4.38 * *$ & $-1.70 * *$ & -2.29 & 89.11 & $-0.41 * *$ \\
\hline 49 & M 31-2A × IS 31651 & $10.55 * *$ & $15.5 * *$ & $9.06 * *$ & $289.94 * *$ & 0.12 \\
\hline \multirow[t]{2}{*}{50} & M 31-2A × IS 32439 & $8.71 * *$ & $13.77 * *$ & $12.31 * *$ & $342.61 * *$ & 0.04 \\
\hline & CD@ $5 \%$ & 2.12 & 1.15 & 2.67 & 123.25 & 0.25 \\
\hline
\end{tabular}

$*$ and $* *$ indicates significant at 5 and 1 per cent, respectively. 
The cross 104A $\times$ IS 31651 (10.54) exhibited highest sca towards negative direction. At the same time 15 crosses noticed positive sca effect among them M 31-2A $\times$ IS 31651 (10.54) accounted high positive sca effect. For plant height, 12 were found to have positive significance. The crosses viz., M 312A $\times$ IS 995 (16.92) and M 31-2A × IS 32439 (16.58) topped the list of hybrids showing positive sca effects for plant height (Table 3).

Among 50 crosses, 12 crosses showed significant for sca effects for number of leaves per plant and out of which six were having positive sca effects. The crosses viz., 104A × IS 19975 (1.54), M 31-2A × IS 29654 (1.45) and 104A $\times$ IS 30451 (1.38) expressed significant positive sca effect. For panicle width, out of 50 hybrids evaluated twenty-six recorded significant sca effects and of these 13 had positive and other 13 had negative significant sca effects. The cross M 31-2A $\times$ IS 32439 (1.37) showed peak significant positive sca effect.

Eight hybrids accounted for significant sca effects for panicle length and of these four had positive and other four had negative sca effects. The hybrids viz., 104A $\times$ IS 19450 (5.03), 104A $\times$ IS 26617 (4.33), M 31-2A $\times$ IS 602 (4.83) and M 31-2A × IS 32439 (3.83) were found to have positive sca effects. Thirty crosses accounted for significant sca effect for number of primaries per panicle of all these 15 crosses showed positive sca effects. The crosses viz., M 31-2A × IS 31651 (10.54) and M 31-2A × IS 32439 (8.71) showed highest positive significant sca effect. In contrast crosses viz., 104 A $\times$ IS 31651 (10.54) and $104 \mathrm{~A} \times$ IS 32439 (8.71) showed highest negative significant sca effects.

For panicle weight, the crosses viz., 104A $\times$ IS 22720 (17.76), 104A × IS 24462 (17.29) and M 31-2A $\times$ IS 19975 (16.63) accounted for highest significant sca effect in positive direction. The values of sca variance for number of seeds per panicle varied from $481.72(\mathrm{M} \mathrm{31-2A} \times \mathrm{IS} 24462)$ to 481.62 (104A $\times$ IS 24462). Among 50 crosses, 17 crosses showed positive and other 17 showed negative significant $s c a$ effects for number of seeds per panicle. The hybrid $104 \mathrm{~A} \times$ IS 24462 (481.62) have shown the highest positive $s c a$ effect and was succeeded by $\mathrm{M}$ 31-2A × IS 11619 (408.27).

Thirty hybrids accounted for significant sca effect for grain yield per plant and of these 15 had positive sca effects. The hybrid M 31-2A $\times$ IS 11619 (16.47) have shown the highest positive effect succeeded by $M$ 31-2A $\times$ IS 32439 (12.31). The hybrid 104 A $\times$ IS 11619 (16.47) had shown the highest negative sca effect which was succeeded by $104 \mathrm{~A} \times$ IS 32439 (12.31). Twenty-six crosses were found to have significant sca effects for 100 seed weight. Out of which the crosses viz., $104 \mathrm{~A} \times \mathrm{IS} 28313(0.44), 104 \mathrm{~A} \times$ IS 30451 (0.41), M 31-2A $\times$ IS 4581 (0.37) and M 31$2 \mathrm{~A} \times \mathrm{IS} 995(0.34)$ recorded highest values for positive $s c a$ effects

It is concluded that the female parent M 312A observed as a good general combiner for plant height, number of leaves per plant, number of primaries per panicle, panicle weight, number of seeds per panicle, grain yield per plant and 100 seed weight. From the studies it was observed that the hybrids viz., M 31-2A $\times$ IS 11619, M 31-2A $\times$ IS 32439, M 31-2A $\times$ IS 31651 and 104A $\times$ IS 24462 were best specific combiners for grain yield per plant. The higher magnitude of SCA variance over GCA variance was observed for all the characters studied except for panicle length which indicates the prevalence of nonadditive gene action for these characters Thus, it can be concluded that both inter and intra allelic interactions were involved in the expression of theses quantitative traits. The parental lines in this study were having 
diverse genetic background of their source populations, and hence their hybrids exhibited high specific combining ability effects.

\section{References}

Dehiwal, A. K., Pahuj, S. K., Shafiqurrahaman, M., Kumar, A. and Sharma, P. 2017. Studies on combining ability for yield and its component traits in forage sorghum, Int. J. Pure App. Biosci., 5(5): 493-502.

Jadhav, R. R. and Deshmukh, D. T. 2017. Heterosis and combining ability in sorghum [Sorghum bicolor (L.) Moench] over the environments. Int. J. Curr. Microbiol. Appl. Sci., 6(10): 3058-3064.

Kempthorne, O. 1957. An introduction to genetic statistics, Jhon wiley sons,
Newyork.

Kumar, S. and Chand, P. 2015. Combining ability and heterosis for grain yield, fodder yield and other agronomic traits in sorghum [Sorghum bicolor (L.) Moench]. J. Appl. Nat. Sci., 7 (2): 1001 $-1005$

Panse, V. G. and Sukhatme, P. V. 1967. Statistical methods for agricultural workers, ICAR, New Delhi.

Reddy, P. S., Patil, J. V., Nirmal, S. V. and Gadakh, S. R. 2013. Improving postrainy season sorghum productivity in medium soils: does ideotype breeding hold a clue. Curr. Sci., 102 (6): 904908.

Sprague G. F. and Tatum L. A. 1942. General and specific combining ability in single crosses of corn. J. Am. Soc. Agron., 34: 923-932.

\section{How to cite this article:}

Sandeep, N., B.D. Biradar, Mruthunjaya C. Wali and Balikai, R.A. 2019. Studies on Combining Ability for Yield and Its Component Traits in Rabi Sorghum [Sorghum bicolor (L.) Moench]. Int.J.Curr.Microbiol.App.Sci. 8(09): 353-364.

doi: https://doi.org/10.20546/ijcmas.2019.809.042 